\title{
Le poète à l'essai : \\ une transaction secrète chez Ralph Waldo Emerson
}

Yves GARDES, Université Paris Dauphine

DOI : https://doi.org/10.3138/cras.2017.027

Dans "The Poet », Ralph Waldo Emerson peint le portrait du "poète véritable " (" true poet " $E L 450$ ), libre, qui contraste en tous points avec les " hommes aux talents poétiques " ("men of poetic talents" $E L 450$ ), enchaînés dans la prison de leur intellect. Tout au long de l'essai, Emerson met un point d'honneur à tourner en ridicule les poètes ordinaires, les poètes traditionnels, dont les exploits littéraires ne survivent pas au temps des mondanités, et leur préfère les vers du poète authentique, du poète à venir, qu'il prophétise dans le même mouvement de l'essai. Dans cette polarisation entre le poète véritable et le poète ordinaire, la voix de l'essai adopte le point de vue d'une personne emprisonnée, au même titre que celle du poète traditionnel. Il s'installe alors un sentiment paradoxal, car, si la voix narrative admet sa captivité (EL 451-452), le poète présenté semble pourtant renvoyer à la voix narrative de l'essai, plus communément appelée Emerson, de sorte que le lecteur semble se tenir en présence de deux voix : celle de l'essai manifeste et lisible, et celle du poète, silencieuse et invisible.

L'enjeu de cet article consiste alors à mettre au jour la " transaction secrète $»^{1}$ qui semble avoir lieu entre la voix de l'essai et celle du poète. Plus précisément, il s'agira de d'explorer dans quelle mesure la voix du poète peut répondre à celle de l'essai, car, si la collision des voix et la transformation réversible de l'une en l'autre n'a pas lieu dans "The Poet », l'espoir de faire advenir une voix poétique dans le champ réflexif du discours normatif est pourtant formulé dans l'exhortation finale au poète de révéler ses chants. (EL 467-468)

Pour ce faire, il conviendra tout d'abord de s'intéresser à la logique discursive de l'essai « The Poet» pour affirmer qu'Emerson ne présente pas directement le poète, mais son négatif photographique, à distance de son incarnation effective dans le discours (première section). Puis il sera question de lire un poème d'Emerson, et de se concentrer sur le moment de crise que traverse le poète dans l'exercice de son art - la conversion de la perception en expression -, pour confirmer que le poète attendu ne peut s'incarner à la manière dont le propose l'essai "The Poet " (deuxième section). Enfin, il faudra s'interroger sur la possibilité même de réconcilier les deux voix identifiées dans «The Poet » et relire un passage de l'essai «Experience " pour montrer que la voix du poète peut parfois s'entrelacer avec celle de l'essai, et ainsi mettre au jour les modalités de cette transaction secrète.

La logique discursive du négatif

Dans Emerson's Romantic Style, Julie Ellison lit dans "The American Scholar" un affrontement entre deux classes antagonistes «us » et "them » - les Américains d'une part, et les Européens d'autre part -, qu'Emerson met en scène par le négatif. (Ellison 98) Dans Emerson and the Conduct of Life, David Robinson souligne quant à lui que l'esprit objectif du photographe ou du portraitiste donne le ton de la série de conférences The Times, en particulier celui de "The Transcendentalist ", conférence centrale dans laquelle Emerson manipule la distance qui sépare la 
voix narrative et le transcendantaliste qu'il décrit. (Robinson 55) Une observation similaire s'applique à "The Poet " - un essai qui trouve le plus clair de son matériau dans la conférence sur le poète de cette même série The Times - car en effet, la construction prototypique du poète véritable semble procéder d'une déconstruction du poète traditionnel en divers mouvements de négation successifs, qui font apparaître par le négatif de l'image traditionnelle du poète, l'essence de celui qu'Emerson appelle de ses vœux. En définissant tout d'abord le poète par ce qu'il n'est pas, Emerson renverse le portrait du poète sur lui-même, à la façon dont un artiste esquisse les premiers contours de sa toile dans une camera obscura avant de la retourner, pour finalement la peindre à partir de cette substance fondamentale.

L'incipit de l'essai se lit tout d'abord comme le diagnostic d'un simulacre culturel contemporain. Emerson peint et dénonce de concert la culture « limitée » de ses contemporains, dont l'étude de "règles" et de "cas particuliers" s'apparente à la mémorisation de répliques d'un "spectacle " qui ne vise d'autre but que celui de " divertir ». La scène culturelle est ainsi tournée en théâtre de dupes, où se jouent inlassablement les mêmes pièces, à distance de l'expérience du réel. Les doctrines prononcées sur le beau ne peuvent être que "superficielles " car les acteurs de ces pièces des "amateurs" - récitent leurs répliques sans jamais les incarner. Les poètes conventionnels se contentent d'une "vie policée et conformiste » et n'écrivent de poèmes que lorsque bon leur semble, sans attacher ou même rattacher la forme de leurs poèmes à l'essence de leur expérience (" at a safe distance from their own experience " EL 447), de sorte que le problème poétique semble procéder d'une inadéquation entre la forme du poème et sa substance.

Le poète véritable est lui tout d'abord présenté comme le négatif de l'homme ordinaire : " [The poet] stands among partial men for the complete man, and apprises us not of his wealth, but of the commonwealth ». (EL 448) Le poète se distingue ainsi par sa position dans la société, dans laquelle il tient pour, c'est-à-dire qu'il représente l'homme complet, tandis que ses contemporains demeurent incomplets. En d'autres termes, le poète n'est pas incomplet. Plus encore, le poète instruit ses contemporains non pas de sa propre richesse - tel serait le cas des "faux " poètes -, mais de la richesse commune. Ainsi le poète s'affirme-t-il par la négation de l'homme ordinaire. Par ailleurs : "He is isolated among his contemporaries, by truth and by his art, but with this consolation in his pursuits, that they will draw men sooner or later ». (EL 448) La préposition " among " agit par deux fois (" He stands among partial men" et " He is isolated among his contemporaries") comme un opérateur de parcours du corps social dans son ensemble et de singularisation du poète en particulier, pour affirmer par-delà l'unité sociale apparente une polarisation entre les hommes ordinaires et le poète.

Après avoir présenté le poète comme ce qu'il n'est pas, Emerson le définit désormais en regard de ce que les hommes ordinaires ne sont pas: "[T]he great majority of men seem to be minors, who have not yet come into possession of their own, or mutes, who cannot report the conversation they have had with nature ». (accentuation personnelle, EL 448) Les propositions négatives qui décrivent les hommes ordinaires, sont à lire en surplomb de la première description négative, de sorte que par double négation apparaissent dans l'esprit du lecteur les premiers traits positifs du poète : le poète est en pleine possession de ses moyens; le poète est en mesure de rapporter la conversation qu'il a entretenue avec la nature. L'intuition du lecteur est même convertie en aspiration plus bas dans le texte : "Every man should be so much an artist, that he could report in conversation what had befallen him ". (accentuation personnelle, EL 448) Ce que le poète est, l'homme ordinaire devrait y aspirer aussi. À travers le destin qu'Emerson lui réserve, le poète acquiert désormais une identité qui s'exprime par le positif: "The poet is the person in whom these powers are in balance, the man 
without impediment, who sees and handles that which others dream of, traverses the whole scale of experience, and is representative of man, in virtue of being the largest power to receive and to impart ». (EL 448) La première véritable description positive du poète se lit ainsi au terme d'un mouvement dialectique non-synthétique de négation, mais elle ne se donne pourtant pas comme une conclusion sur l'identité du poète, car l'essai fait immédiatement retour au négatif.

En effet, la logique discursive de l'essai consiste en une oscillation constante entre ce que le poète n'est pas et ce que les hommes ordinaires ne sont pas, suggérant dans le même geste ce que le poète est. En d'autres termes, le poète n'est pas, mais il est: "Therefore the poet is not any permissive potentate, but is emperor in his own right ". (accentuation personnelle, EL 449) Ou encore : "The Poet does not wait for the hero or the sage, but [...] he writes primarily what will and must be spoken ». (accentuation personnelle, EL 449) La logique discursive de l'essai oscille constamment entre la négation de ce qu'est le poète et l'affirmation de ses traits caractéristiques pour souligner la distance qui sépare le poète de l'homme ordinaire. Il s'en suit que le texte œuvre à la formulation du paradoxe inhérent à l'entreprise émersonienne : le poète véritable n'est pas accessible, mais il est dans le même temps un homme représentatif qui devrait inspirer ses contemporains.

Dans son martellement d'une scission irrémédiable entre ce que l'intellect collectif conçoit comme poète et celui qu'Emerson appelle de ses vœux, l'essai reprend à son compte le mythe d'Icare pour souligner le contraste entre le poète ordinaire et le poète véritable. Dans un premier extrait, Emerson relate le plaisir avec lequel il débute la lecture d'un poème, et les espoirs qu'il nourrit de "briser ses chaînes " (EL 451) pour s'élever vers l'éther ( $E L$ 452), avant que ses attentes ne soient déçues :

Oftener it falls, that this winged man, who will carry me into the heaven, whirls me into the clouds, then leaps and frisks about with me from cloud to cloud, still affirming that he is bound heavenward; and I, being myself a novice, am slow in perceiving that he does not know the way into the heavens, and is merely bent that I should admire his skill to rise, like a fowl or a flying fish, a little way from the ground or the water; but the all-piercing, all-feeding, and ocular air of heaven, that man shall never inhabit. I tumble down again soon into my old nooks, and lead the life of exaggerations as before, and have lost faith in the possibility of any guide who can lead me thither where I would be. (EL 452)

Tel Icare volant vers le soleil, la voix narrative de "The Poet " aspire à s'élever vers les cieux, mais les révolutions aériennes majestueuses se transforment en simples sauts d'exocet, de sorte que la voix «I " est renvoyée à la condition d'homme inachevé. À l'inverse, dans un autre extrait, les ailes ne se font plus le symbole d'une chute vertigineuse vers le bas et le commun, mais plutôt celui d'une ascension de l'âme du poète :

These wings are the beauty of the poet's soul. The songs, thus flying immortal from their mortal parent, are pursued by clamorous flights of censures, which swarm in far greater numbers, and threaten to devour them; but these last are not winged. At the end of a very short leap they fall plump down, and rot, having received from the souls out of which they came no beautiful wings. But the melodies of the poet ascend, and leap, and pierce into the deeps of infinite time. (EL 458)

Ces deux extraits suggèrent, par les trajectoires divergentes qu'ils suivent, deux voix diamétralement opposées. La première, celle de l'essai et portée par le pronom "I", est celle de l'homme ordinaire 
auquel le lecteur est tenu de s'identifier. La seconde, celle du poète, propose en revanche de conjurer le sort réservé à Icare et de parvenir à une puissance d'élévation interdite à la première voix. Si elles s'affrontent, ces deux voix n'entrent pourtant jamais en collision, de sorte que la conversation entretenue semble prendre l'allure d'un dialogue de sourds.

Les négations répétées tout au long de l'essai n'attestent pas seulement du degré d'abstraction avec lequel Emerson envisage le poète en regard de l'homme ordinaire, elles conduisent aussi le lecteur à s'interroger sur la possibilité même de l'existence d'un tel poète, en dépit de la confiance affichée par Emerson. Intéressé par cette question dans The Liberating Gods (1971), John Q. Anderson en conclut qu'aucun des poètes connus d'Emerson ne remplit les critères d'élection - pas même Milton, ${ }^{2}$ pas même Shakespeare, ${ }^{3}$ pas même Whitman. ${ }^{4}$ Le poète émersonien apparaît ainsi telle une abstraction qui excède tout poète vivant ou ayant existé, qu'il s'agisse d'un simple homme au talent poétique ("men of poetical talents"EL 450) ou Shakespeare. À ce titre, la relation qu'Emerson entretient avec l'œuvre de Whitman illustre bien l'ambiguïté entre la voix narrative de "The Poet ", prise dans l'étau de l'intellect, et celle du poète, libre et silencieuse, mais bel et bien palpable. Il est ainsi étonnant de remarquer que l'estime en laquelle Emerson tient tout d'abord Whitman dans sa célèbre lettre de 1855, n'est soutenue par aucune prise de position ultérieure. Tandis qu'Emerson use tout d'abord de superlatifs pour décrire Leaves of Grass comme l'œuvre la plus extraordinaire jamais produite en Amérique, (PP 563) le Sage de Concord n'inclut pas Whitman dans l'anthologie de poèmes qu'il publie en 1874 sous le titre de Parnassus. L'oscillation entre l'éloge et le silence rend, dans ce cas, bien compte de la duplicité de la voix qui gouverne "The Poet ". Car il survit à la conclusion de John Q. Anderson l'intuition que la voix de l'essai en sait beaucoup sur le poète, ou du moins, en sait assez pour le décrire. Cela paraît d'autant plus surprenant que la voix de l'essai se présente elle-même comme un "novice » $(E L$ 452) dont l'existence est menée " dans les nuages et les airs opaques " (EL 451), et dont " les chaînes sont destinées à être rompues " (EL 451). Quelle légitimité possède donc cette voix à évoquer le poète ? Il s'agit là d'un paradoxe que Sharon Cameron relate en ces termes :

$[\mathrm{O}] \mathrm{n}$ the one hand, the reference for the speaking voice is the unemancipated person who anticipates the poet. But, on the other, the poet being evoked also seemed referenced to the subject-position we call Emerson. The one who calls for the poet, who calls the poet forth, is the one who knows enough of bondage not to be wholly or even mainly defined by freedom. Thus the essay charts two positions and has a double voice. ("The Way of Life " 27)

Dans l'économie de son essai, Sharon Cameron en conclut que "The Poet" ne fournit pas les conditions dans lesquelles le personnel se transforme en impersonnel. Une telle remarque invite pourtant à s'étendre plus longuement sur les termes de la négociation entre la voix de l'essai et la voix silencieuse du poète, et à s'interroger sur les termes de cette "transaction secrète". Mais avant d'explorer la relation qu'entretiennent ces deux voix, il convient de préciser, en relisant un poème oublié d'Emerson, dans quelle mesure le poète décrit dans l'essai ne constitue pas tant un modèle à incarner qu'un poète idéal et inapprochable, car les traits majeurs qui le définissent - la perception et l'expression - et leur articulation dans la pratique poétique s'avèrent un rouage essentiel de la stratégie émersonienne. 
De la crise poétique : perception contre expression

"The Discontented Poet: A Masque » est un poème qui interroge les dons poétiques sur fond de scepticisme. Ce poème, dont la première mouture date de 1838 , ne fut jamais publié, car Emerson n'eut de cesse, tout au long de sa carrière littéraire, d'ajouter quelque deux cents vers, de changer de titre, et de modifier la structure. Avec le temps, "The Discontented Poet: A Masque " devint "The Poet ", un poème divisé en deux sections intitulées "Fragments on the Poet and the Poetic Gift » et "Fragments on Nature and Life ". Cette dernière version fut publiée dans l'édition centennaire de 1904, tandis que la première version de 1838 ne réapparut qu'en 1986 dans The Poetry Notebooks. Aussi est-il intéressant de lire ce poème contre l'essai "The Poet ", tant le destin réservé à ces deux textes - un célèbre essai d'une part et un poème oublié d'autre part - reflète la substance de leur discours. Tandis que "The Poet» célèbre le poète nouveau et encore inapprochable, "The Discontented Poet " peint le portrait d'un homme impuissant, qui éprouve les plus grandes peines à trouver son expression. Le poème souligne ainsi l'insatisfaction qui résulte de la pratique poétique :

His loves were sharp sharp pains

Outlets to his thoughts were none

A wandering fire within his veins

His soul was smouldered \& undone

A cripple of God, half true, half formed,

And by great sparks Promethan warmed

Constrained by impotence to adjourn

To infinite time his eager turn,

His lot of action from the Urn. (CPT 371-372)

David Porter lit ce poème comme le moment de crise qu'Emerson traverse dans sa carrière poétique, et comme un aveu sur les limites de son talent poétique. (Porter 119) Mais il est tout autant possible de faire l'économie d'une équation entre la voix du poème et Emerson lui-même, pour s'intéresser au moment de crise poétique en tant que tel, sans faire retour au référent de la voix poétique. Dans cette deuxième strophe, le poète est réifié en position d'objet, par l'énumération de ses possessions immatérielles: "His loves", " his thoughts ", " His soul ", " his eager turn ", " His lot of action ». Certaines d'entre elles sont niées ("His soul was smouldered \& undone »), tandis que d'autres sont transformées en leur opposé ("His loves were sharp sharp pains»), mais toutes pointent vers la paralysie du poète et soulignent l'échec de son expression en tant qu'individu. La répétition de l'adjectif "sharp " souligne lui les peines endurées par le poète et suggère dans le même geste le bégaiement premier de l'expression poétique que confirme le reste de la strophe et sa syntaxe heurtée.

Le poème interroge la possibilité même d'incarner le poète présenté dans l'essai : est-il seulement possible de représenter l'homme complet? La réponse se lit plus loin dans le poème, dans une strophe où le poète se désespère de convertir le chant des muses :

I see your forms with deep content

I know that ye are excellent;

But will ye stay?

I hear the rustle of wings

Ye meditate what to say 
When ye go to quit me forever \& aye. (CPT 372)

Dans les deux premiers vers de cette strophe, le poète prend à sa charge la perception des formes ( I see »), mais laisse aux muses la responsabilité de les exprimer ("Ye meditate what to say »). La distance qui sépare la perception de l'expression se mesure ainsi à l'aune d'un doute, formulé dans une question adressée aux muses, qui, plus que de donner des formes à exprimer, les donnent à voir. Le poète n'en paraît que plus ordinaire et incomplet, car il manque d'exercer son pouvoir expressif dans le même temps que son pouvoir perceptif. De plus, si les muses apaisent les craintes du poète dans la strophe suivante, le jugement qu'elles formulent à son encontre n'en est que plus sceptique : «From thyself thou canst not flee, / From thyself no more can we ". (CPT 373) Si les muses n'abandonnent pas le poète, le poète ne peut, lui, pas abandonner sa condition incomplète d'homme et représenter l'homme complet de l'essai. "The Discontented Poet " n'est autre qu'un poète ordinaire, un simple "homme au talent poétique " qui ne s'exprime pas mieux qu'en rimes plates, naïvement agencées de façon à imiter un sonnet shakespearien sans pourtant y parvenir (" pains ", " none ", "veins ", " undone », " formed ", " warmed ", " adjourn ", " turn ", " Urn »).

Pour autant, le spectre de "veins" du poème ("A wandering fire within his veins / His soul was smouldered \& undone ») résonne dans l'homophone "vain» de la célèbre formule de l'essai («I look in vain for the poet whom I describe " $E L$ 465), de sorte que la vanité de la quête du poète se lit désormais comme la recherche éperdue du poète en sommeil chez l'essayiste. La distance qui sépare la voix de l'essayiste de celle du poète dans leur transaction secrète ne procède donc pas tant d'une mesure spatiale que d'une mesure temporelle, d'un retard entre la reconnaissance de l'existence du poète et la prise en charge de cette existence par un sujet poétique - un retard dont Thoreau atteste dans son poème "The Poet's Delay ":

In vain I see the morning rise,

In vain observe the western blaze,

Who idly look to other skies,

Expecting life by other ways.

Amidst such boundless wealth without,

I only still am poor within,

The birds have sung their summer out,

But still my spring does not begin.

Shall I then wait the autumn wind,

Compelled to seek a milder day,

And leave no curious nest behind,

No woods still echoing to my lay? (Thoreau 535-536)

Si la perception des phénomènes de la nature paraît vaine ("In vain I see », "In vain observe ») et n'enrichit pas de prime abord le poète ("I only still am poor within »), c'est parce que l'expérience immédiate des trésors de la nature se suffit à elle-même - quel besoin d'expression si le spectacle naturel se déploie sous le regard du poète ? Dans le temps de la perception, l'expérience se diffuse pourtant dans les veines du poète qui, au terme d'un retard consécutif à une saison contrariée ("But still my spring does not begin. / Shall I wait the autumn wind, "), synonyme de privation de l'expérience, sera en mesure de la reformuler par le langage, et de restituer le sentiment de désarroi qui animait le poète dans l'instant de la perception - l'esthétique apollinienne est seulement 
consécutive, et non concomitante, à l'ivresse dionysiaque. Comme le souligne Mathieu Duplay, «la création poétique est indissociable d'une forme de temporalité ; représentante d'un idéal auquel la réalité est infidèle, la poésie crée la possibilité d'une histoire, elle crée du mouvement par lequel ce qui est tend à se rapprocher de ce qui doit être ». (Duplay 121)

Chez Emerson, "The Discontented Poet " manque d'incarner le poète célébré dans l'essai car il manque d'exercer en même temps ses deux offices de "Seer " et " Namer ». Soit il est "Seer ", soit il est "Namer ". Ou plus précisément, il est tout d'abord «Seer », et "Namer " seulement ensuite. Bien qu'il soit un poète, il reprend le rôle d'homme incomplet et n'échappe pas à sa nature partielle. En fin de compte, le temps arbitre entre le poète idéal et le poète ordinaire selon les termes du principe organique cher à Emerson (EL 458) : il faut du temps pour que ce qui est perçu soit assimilé, métabolisé par le poète, qui alors seulement sera en mesure de donner une expression à son expérience. Il faut ainsi en conclure que le poète qu'Emerson recherche en vain n'est peut-être pas tant le jeune poète prodige qui assumerait les deux offices simultanément dans sa pratique poétique, mais une manière de réconcilier les différents temps de la pratique poétique dans un même geste d'écriture. Il convient alors de faire retour à la "voix double" identifiée par Sharon Cameron, car les essais fournissent les conditions idéales pour qu'affleure à la surface du texte une transaction entre une voix narrative qui porte le sceau de la perception et une voix poétique qui porte celui de l'expression.

La voix poétique : un « I » intempestif

Rechercher des occurrences où la voix narrative des essais et celle du poète idéal se rencontrent doit ainsi comprendre dans quelle mesure leur transaction permet d'accomplir le défi lancé par l'essai «The Poet ». C'est d'ailleurs la voie que Stanley Cavell propose indirectement de suivre dans un passage de "Thinking of Emerson " où le philosophe établit une analogie entre la relation qu'Emerson cultive avec sa propre écriture, et celle que Heidegger noue avec la poésie de Hölderlin :

Emerson's [...] relation to poetry is inherently [his] interest in [his] own writing; [he is his] own Hölderlin. I do not mean [his interest] in what we may call [his] poems, but [his] interest in the fact that what [he is] building is writing, that [his] writing is, as it realizes itself daily under [his] hands, sentence by shunning sentence, the accomplishment of inhabitation, the making of it happen, the poetry of it. (Cavell 131)

La transaction qui a court entre le « construire » et l' " écrire » doit s'entendre comme une transaction entre la voix du poète - celle qui crée, qui construit le langage - et celle de l'essayiste - celle qui écrit. De la même manière, "l'accomplissement de l'habitation " s'entend comme un "habiter poétique " au sein d'un Parnasse poétique, demeure des muses, où la voix de l'essai s'ouvre à celle du poète. Il s'agira donc ici de soutenir qu'une voix poétique, différente de la voix narrative des essais, surgit çà et là dans les apories du discours logique et normatif.

Pour ce faire, il convient de relire un passage d' "Experience", essai que Julie Ellison considère à raison comme le compagnon de "The Poet». ("Tears for Emerson » 161) Mais il ne sera pas tant question de faire écho à la lecture de Sharon Cameron, que de proposer une approche sémiotique influencée par Julia Kristeva, pour proposer une lecture parallèle du célèbre passage sur le chagrin : 
The only thing that grief has taught me, is to know how shallow it is. [...] Grief too will make us idealists. In the death of my son, now more than two years ago, I seem to have lost a beautiful estate-no more. I cannot get it nearer to me. [...] This calamity [...] does not touch me: some thing which I fancied was part of me, which could not be torn away without tearing me, nor enlarged without enriching me, falls from me, and leaves me no scar. I grieve that grief can teach me nothing. (EL 472-473)

L'étonnement que la dernière phrase «I grieve that grief can teach me nothing "soulève chez le lecteur s'explique par la contradiction que l'énoncé semble receler. La proposition relative suggère que l'expérience du chagrin ne constitue pas une source de connaissance. Par voie de conséquence, la validité même de cette expérience est mise à mal. Selon les termes de cette proposition, le sujet, placé dans en position d'objet "me ", est donc bien en mal de faire l'expérience du chagrin, et contredit ainsi la proposition principale, et la capacité du sujet «I » à faire l'expérience du chagrin. L'esprit du lecteur corrige spontanément ce paradoxe, en admettant, avec Emerson, que la seule expérience possible du chagrin est celle de sa futilité ("shallowness »). "I » et " me » renvoient ainsi à la même voix et la logique du discours est résorbée. Mais la proposition relative n'affirme pas "grief can teach me nothing but one ", mais bel et bien "nothing", de sorte que "I» et "me " ne semblent pas renvoyer à la même voix. Si la connaissance du chagrin est niée pour "me », comment se peut-il que ce même "me ", désormais placé en position de sujet "I ", reconnaisse le chagrin comme précisément l'objet de son expérience ? Pour résoudre ce paradoxe, il faut considérer que deux voix sont à l'œuvre dans la même phrase, par-delà l'identité apparente de "me " et "I ». Mais si l'on attribue l'une des ces voix à celle de l'essai, plus communément appelée Emerson, à qui appartient cette deuxième voix?

Deux types de pratique signifiantes semblent à l'œuvre: la première pratique, celle de la parole, et la seconde, celle de l'énoncé :

On parle lorsqu'on juge, donc lorsqu'on adopte la logique de la parole (le Logos), et alors la négation comme attitude interne au jugement se présente sous la forme de la loi $\mathrm{du}$ tiers exclu. On énonce lorsque dans une démarche de négativité (de différenciation) on englobe dans l'acte de la signification ce qui n'a pas d'existence dans la logique (la parole) et qui est le terme nié (=point de départ de la signification). (Kristeva 189)

L'énoncé accomplit cette pratique signifiante dans laquelle la négation ne suit pas la logique de la parole, quand "cette 'négation' affirme ce qui est nié dans un geste non plus de jugement (tel est le geste de la parole) ». (Kristeva 190) Les énoncés poétiques illustrent parfaitement ce type de pratique signifiante, parce qu'ils effectuent une «mise à jour de la production signifiante, ce geste qui réunit simultanément le positif et le négatif, ce qui existe pour la parole et ce qui est non-existant pour elle ». (Kristeva 190) Ainsi, " la signifié poétique jouit d'un statut ambivalent: il est à la fois (donc en même temps, et non successivement) concret et général». (Kristeva 191) Sans parvenir à la synthèse, le signifié poétique réunit le concret et le général et rejette l'individualisation, de sorte que l'unité du discours se diffracte en deux voix qui maintiennent l'énoncé en tension constante entre le concret et le général - le lecteur est bien incapable d'identifier clairement à qui renvoient "I " et "me ", même si les deux pronoms semblent alternativement renvoyer à Emerson. Et c'est bien là le propre du signifié poétique, car il « renvoie et ne renvoie pas à un référent; il existe et n'existe pas, il est en même temps un être et un non-être ». (Kristeva 192) 
Si l'on admet que la voix de "me», la logique impose de laisser vacante la place que devrait occuper le sujet du prédicat " grieve » de la proposition principale. Mais cette place est occupée par le sujet «I ", un "I » intempestif qui s'immisce dans l'aporie du discours logique. Par l'affirmation "I grieve ", "I " nie la logique dans laquelle il s'inscrit pourtant (" grief can teach me nothing »). Ce sujet est appelé "zérologique ", car c'est " un non-sujet qui vient assumer cette pensée qui s'annule ». (Kristeva 212) En d'autres termes, ce sujet zérologique autorise l'expression du signifié poétique : il peut se lire comme un sujet poétique qui ne possède pas de référent précis, ou plutôt, qui possède une multitude de référents potentiels. Le sujet zérologique surgit donc dans le temps de l'expression ("I grieve") consécutif au temps de la perception, de l'observation et de l'analyse que le chagrin n'enseigne rien ("grief can teach me nothing »). Par conséquent, le sujet zérologique se lit comme la voix du poète encore inapprochable, qui réussit là où le poète ordinaire échoue, car il permet, dans l'espace d'une même phrase, la réconciliation de la perception et de l'expression.

La recherche du sujet zérologique par le geste de l'écriture s'apparente ainsi à l'abandon à l'impersonnel prôné par Emerson dans "Circles " $E L 414)$, car l'éclipse momentanée de la logique de la parole fait dans le même geste s'effacer le sujet, qui reparaît, de façon brutale, mais aussi par nécessité, métamorphosé et déshabillé de ses traits personnels. Si le poète est le "praticien de l'impersonnel» ("The Way of Life» 4) qui pourtant n'affleure pas dans "The Poet», le sujet zérologique surgit lui dans "Experience " et donne à lire une mise en œuvre du dispositif annoncé par Emerson. Cette occurrence du sujet zérologique ne constitue d'ailleurs pas un cas isolé et se retrouve dans de nombreux essais, et assez célèbrement dans le passage de Nature sur le globe oculaire: "Standing on the bare ground,-my head bathed by the blithe air, and uplifted into space,-all mean egotism vanishes. I become a transparent eye-ball; I am nothing; I see all; the currents of the Universal Being circulate through me; I am part or particle of God ». (EL 10) Une nouvelle fois, la négation du sujet «I " ( I am nothing ») impose à la logique de laisser vacante la place pourtant occupée par un sujet "I» ("I see all»), reconstitué dans le geste poétique de l'écriture.5 Le devenir "globe oculaire transparent" semble d'ailleurs anticiper le devenir " impersonnel " de Mallarmé qui, dans une lettre adressée à Cazalis, l'exprime en termes pour le moins émersoniens :

J'avoue du reste, mais à toi seul, que j'ai encore besoin, tant ont été grandes les avanies de mon triomphe, de me regarder dans cette glace pour penser, et que si elle n'était pas devant la table où je t'écris cette lettre, je redeviendrais le Néant. C'est t'apprendre que je suis maintenant impersonnel, et non plus Stéphane que tu as connu, mais une aptitude qu'a l'Univers Spirituel à se voir et à se développer, à travers ce qui fut moi.

(Mallarmé 78)

L'abandon à l'impersonnel prend l'allure d'un abandon au sujet zérologique, qui, affranchi du carcan de la normalité logique, s'immisce dans les apories du discours. Le dialogue de sourds qui s'installe souvent entre deux voix qui cohabitent pourtant au sein de l'espace textuel émersonien procède in fine de cette transaction secrète, de cette conversation entre la voix narrative et la voix poétique des essais. Cette transaction permet ainsi à Emerson-poète de venir en aide à Emerson-essayiste : "I think that philosophy is still rude and elementary. It will one day be taught by poets ». (W12:13)

Une telle position sur le poète trouve son origine dans la tradition romantique allemande, notamment chez Novalis, dont le commentaire sur la transaction entre philosophie et poésie éclaire la sentence émersonienne : 
La poésie élève chaque individu à la totalité à travers une opération de connexion qui lui est propre - et si la philosophie, par sa jurisprudence, prépare le monde à l'influence des idées, alors la poésie est pour ainsi dire la clé de la philosophie, son but et son sens. Car la poésie fonde la belle société - la famille universelle - la belle ordonnance de l'univers. Pendant que la philosophie augmente les forces de l'individu à travers le Système et l'État, en lui communiquant les forces de l'humanité et de l'univers, et en transformant ainsi la totalité en organe de l'individu, et l'individu en organe de la totalité, la poésie réalise la même opération au niveau de la vie. L'individu vit dans la totalité et la totalité dans l'individu. La poésie engendre la sympathie supérieure et la coactivité, la communion intime du fini et de l'infini. (Novalis 55)

Selon Novalis, le but de toute activité philosophique est d'advenir poésie. Philosophie et poésie procèdent en fin de compte d'un même exercice, qui vise une spirale transactionnelle entre l'individu et la totalité. Pourtant, le geste philosophique ne saurait suffire à lui-même, car le discours qu'il produit s'extrait du mode d'existence individuelle. S'il ne parvient pas à s'armer de la "force esthétique " qui anime le poète, l'homme ne sera jamais plus qu'un simple "philosophe de la lettre ». (Schelling 10)

L'abandon de soi à l'impersonnel vise en fin de compte à ébranler l'édifice d'un "moi " sédentaire pour lui donner les possibilités d'une mode d'existence nomade, et constitue la stratégie privilégiée pour affranchir la pensée du carcan de la tradition. Emerson érige ainsi le poète en véritable praticien de l'impersonnel, et, au cour des essais, l'apparition d'un sujet zérologique confirme la mise en ouvre de cette stratégie. Ce sujet zérologique permet ainsi de revigorer le discours logique de la force esthétique du poète, à force de transactions secrètes infinies.

Bibliographie

ANDERSON, John Q. The Liberating Gods: Emerson on Poets and Poetry, Coral Gables: University of Miami Press, 1971.

CAMERON, Sharon. "Representing Grief: Emerson's "Experience.” "Representations 15, 1986. 1541.

-. "The Way of Life by Abandonment: Emerson's Impersonal. " Critical Inquiry, vol. 25, $\mathrm{n}^{\circ} 1$, automne 1998. 1-31. Repris dans Impersonality. Chicago: The University of Chicago Press, 2007. 79-107. Encore repris dans The Other Emerson. Ed. Branka Arsić et Cary Wolfe. Minneapolis: University of Minnesota Press, 2010.

CAVELL, Stanley. "Thinking of Emerson", in The Senses of Walden. Chicago: The University of Chicago Press, 1992 [1981].

DUPLAY, Mathieu. "Emerson, l'art, le poète ». L'exuvre en prose de Ralph Waldo Emerson. Ed. François Brunet et Anne Wicke. Paris: Armand Colin, 2003. 114-129.

ELLISON, Julie. Emerson's Romantic Style. Princeton: Princeton University Press, 1984.

-. "Tears for Emerson: Essays, Second Series." The Cambridge Companion to Ralph Waldo Emerson.

Ed. Joel Porte et Saundra Morris. Cambridge: Cambridge University Press, 1999. 140-161.

EMERSON, Ralph Waldo. Essays and Lectures. Ed. Joel Porte. New York: Library of America, 1983.

- Collected Poems and Translations. Ed. Harold Bloom et Paul Kane. New York: Library of America, 1994. 
-. The Early Lectures of Ralph Waldo Emerson, Vol. III, 1838-1842. Ed. Robert E. Spiller et Wallace E. Williams. Cambridge: The Belknap Press of Harvard University Press, 1972.

-. The Works of Ralph Waldo Emerson. 12 vols. New York: Fireside Edition, 1909

-. Collected Works of Ralph Waldo Emerson, volume IX: Poems - A Varorium Edition. Ed. Albert J. von Frank et Thomas Wortham. Cambridge: The Belknap Press of Harvard University Press, 2011.

—. Emerson's Prose and Poetry. Ed. Joel Porte et Saundra Morris. New York: Norton Critical Edition, 2001.

GrimSTAD, Paul. "Emerson's Adjacencies: Radical Empiricism in Nature ». The Other Emerson.

Ed.Branka Arsić et Cary Wolfe. Minneapolis: University of Minnesota Press, 2010. 251-270.

KRISTEVA, Julia. Sèméiotikè. Recherches pour une sémanalyse. Paris: Editions du Seuil, 1969.

Mallarmé, Stéphane. Propos sur la poésie. Monaco: Ed. Du Rocher, 1946.

Novalis. Poésie, réel absolu. Paris: Poesis, 2015.

PORTER, David. Emerson and Literary Change. Cambridge: Harvard University Press, 1978.

RoBINSON, David M. Emerson and the Conduct of Life: Pragmatism and Ethical Purpose in the Later

Works. Cambridge: Cambridge University Press, 1993.

SCHELling, Friedrich Wilhelm. Textes esthétiques. Paris: Klincksieck, 2005.

ThOREAU, Henry David. Collected Essays and Poems. Ed. Elizabeth Hall Witherell. New York:

Library of America, 2001.

WoOLF, Virginia. Orlando. London: Vintage, 2004.

${ }^{1}$ L'expression de " transaction secrète » est ici empruntée à Virginia Woolf, qui interroge le définition même de l'écriture poétique en ces termes: "Was not writing poetry a secret transaction, a voice answering a voice? » (Woolf 213)

${ }^{2}$ "But when we adhere to the ideal of the poet, we have our difficulties even with Milton and Homer. Milton is too literary, and Homer too literal and historical. » (EL 465-466)

${ }^{3}$ « He rested in their beauty; and never took the step which seemed inevitable to such genius, namely to explore the virtue which resides in these symbols and imparts this power. " (EL 725)

${ }^{4}$ " Determining Emerson's opinion of Whitman's poetry is the most difficult of all for lack of positive information. Emerson's enthusiasm for Leaves of Grass [...] makes the temptation very great to credit Emerson with a more complete approval of Whitman than he specifically gave. " (Anderson 87)

${ }^{5}$ Pour une lecture approfondie de ce passage, construite sur les modalités d'interactions linguistiques entre les termes, voir "Emerson’s Adjacencies: Radical Empiricism in Nature» de Paul Grimstad. 